\title{
Bicone-like ZnO structure as high-performance butanone sensor
}

\author{
Cecilia A. Zito, Tarcísio M. Perfecto, Taís N.T. Oliveira, Diogo P. Volanti* \\ Laboratory of Materials for Sustainability (LabMatSus), Ibilce, São Paulo State University (Unesp), R. Cristóvão Colombo, 2265, S. J. Rio Preto, SP 15054-000, Brazil
}

\section{A R T I C L E I N F O}

\section{Article history:}

Received 19 February 2018

Received in revised form 28 March 2018

Accepted 5 April 2018

Available online 6 April 2018

\section{Keywords:}

Sensors

Semiconductors

Template-free

Volatile organic compounds

Zinc oxide

\begin{abstract}
A B S T R A C T
Sensors based on metal oxide semiconductors have been widely applied for the detection of several volatile organic compounds (VOCs). However, the development of sensing materials with high selectivity, improved sensitivity, low detection limit is still a challenge. In this work, bicone-like ZnO structure was synthesized by a rapid template-free microwave-assisted hydrothermal method and then applied as VOC sensor. The VOC-sensing tests revealed that $\mathrm{ZnO}$ structure presented a higher response toward butanone than to other VOCs, such as benzene, toluene, $m$-xylene, acetone, ethanol, acetaldehyde, and isopropanol. Moreover, the $\mathrm{ZnO}$-based sensor showed an enhanced butanone-sensing performance at $400{ }^{\circ} \mathrm{C}$, including a response of $29.4-100 \mathrm{ppm}$ of butanone, high sensitivity to detect low butanone concentration ( $2 \mathrm{ppm})$, and a low detection limit of $0.41 \mathrm{ppm}$. Therefore, the unique bicone-like $\mathrm{ZnO}$ structure might be a promising candidate for butanone sensors.
\end{abstract}

(c) 2018 Elsevier B.V. All rights reserved.

\section{Introduction}

The development of volatile organic compounds (VOCs) sensor has attracted considerable attention for air-quality monitoring and disease diagnosis $[1,2]$. Some VOCs in exhaled breath are known to be biomarkers for different diseases [3], for example, butanone and toluene might be related to gastric and lung cancer [4,5] respectively, while acetone is associated to diabetes [6]. Thus, VOC sensors with sensitivity enough to detect low ppm (parts per million) are required.

Metal oxide semiconductors (MOS) have been widely used as chemiresistive sensors for VOCs detection, due to its easy production, low cost, and simplicity of use $[7,8]$. However, the production of sensors with high selectivity, sensitivity, and low detection limit is still a challenge. Among the MOS, zinc oxide ( $\mathrm{ZnO})$, an n-type MOS, has demonstrated a great potential for the detection of several VOCs, including acetone [9], ethanol [10], acetaldehyde [11], formaldehyde [12], triethylamine [13], and so on. Nevertheless, to the best of our knowledge, there has been still no reports of $\mathrm{ZnO}$ as a chemiresistive sensor for butanone vapor.

In this paper, bicone-like $\mathrm{ZnO}$ structure was prepared by a template-free microwave-assisted (MAH) method and the VOCsensing performance was presented. Due to its unique structure, the $\mathrm{ZnO}$-based sensor exhibited high butanone sensing performance regarding sensitivity, selectivity, and low detection limit.

\footnotetext{
* Corresponding author.

E-mail address: volanti@ibilce.unesp.br (D.P. Volanti).
}

\section{Material and methods}

The bicone-like $\mathrm{ZnO}$ structure was prepared by a template-free MAH method. First, $2 \mathrm{mmol}$ of $\mathrm{Zn}\left(\mathrm{CH}_{3} \mathrm{COO}\right)_{2} \cdot 2 \mathrm{H}_{2} \mathrm{O}$ (Sigma-Aldrich, $\geq 98 \%$ ) was dissolved in $70 \mathrm{~mL}$ of deionized water. Then, $280 \mu \mathrm{L}$ of $\mathrm{NH}_{4} \mathrm{OH}$ (Sigma-Aldrich, 28.0-30.0\% $\mathrm{NH}_{3}$ basis) was added with stirring until complete homogeneity. The final solution was transferred to polytetrafluoroethylene autoclave, sealed, and heated at $140{ }^{\circ} \mathrm{C}$ for $1 \mathrm{~h}$ in a microwave system $(2.45 \mathrm{GHz} / 800 \mathrm{~W})$. After the heating, the autoclave was naturally cooled down, and the product was centrifuged and washed with deionized water and ethanol, then dried at $80^{\circ} \mathrm{C}$

The sample was characterized by X-ray diffraction (XRD, Rigaku MiniFlex 300), field-emission scanning electron microscopy (FESEM, JEOL JSM-7500F), and X-ray photoelectron spectroscopy (XPS, Thermo Scientific K-Alpha X-ray Photoelectron Spectrometer). The XPS spectra were calibrated using the $C 1$ s peak ( 284.8 $\mathrm{eV})$. Specific surface area was measured by the Brunauer-Emme t-Teller (BET) method through nitrogen physisorption (Gemini VII - Surface Area and Porosity analyzer).

The dispersion prepared by mixing $4.8 \mathrm{mg}$ of the sample with 1 $\mathrm{mL}$ of isopropanol was dropped into alumina substrates with interdigitated gold arrays as electrodes. The as-prepared sensor was dried at $250^{\circ} \mathrm{C}$ for $1 \mathrm{~h}$. The sensor was placed inside the test chamber and heated at the desired operating temperature. The electrical resistance variations were measured after VOCs exposure using a high-voltage source-measure unit (Keithley SourceMeter 2400), applying a voltage of $5 \mathrm{~V}$. The VOCs were injected into the test chamber with a syringe, and then the chamber was cleaned using 
an air flow of $250 \mathrm{~mL} \mathrm{~min}^{-1}$. The sensor response was defined as the ratio $R_{a} / R_{g}$, where $R_{a}$ and $R_{g}$ is the resistance in air and after VOCs exposure, respectively.

\section{Results and discussion}

The morphology of the sample was determined by FESEM. The FESEM image (Fig. 1a) revealed the formation of a bicone-like $\mathrm{ZnO}$ structure, which means that each structure is composed of two cones. The bicone structure presents an average length of 1 $\mu \mathrm{m}$ and a diameter of $\sim 400 \mathrm{~nm}$. The high-magnification FESEM image (Fig. 1b) shows in detail the boundary interface between the two cones. It can be noticed that smaller particles are assembled, acting as building blocks to form the bicones. As shown in Fig. 1c, the energy dispersive X-ray (EDS) spectrum revealed the presence of only $\mathrm{Zn}$ and $\mathrm{O}$ elements, confirming the absence of impurities. According to the XRD pattern of bicone-like $\mathrm{ZnO}$ structure (Fig. 1d), the diffraction peaks could be indexed to the hexagonal ZnO wurtzite structure (JCPDS 36-1451; space group $\mathrm{P6}_{3} \mathrm{mc}$ (1 86 ), $a=3.249 \AA, c=5.206 \AA$ ). No secondary phase was observed, indicating the high purity of the prepared sample. The bicone-like $\mathrm{ZnO}$ structure presented a specific surface area of $21 \mathrm{~m}^{2} \mathrm{~g}^{-1}$.

The chemical composition of the sample's surface was determined by XPS. The high-resolution XPS spectrum (Fig. 2a) of Zn 2p displays two symmetric peaks at $\sim 1021.2 \mathrm{eV}$ and $1044.3 \mathrm{eV}$ related to $\mathrm{Zn} 2 \mathrm{p}_{3 / 2}$ and $2 \mathrm{p}_{1 / 2}$, respectively, with the splitting energy of the two peaks was of $23.1 \mathrm{eV}$, indicating the single oxidation state of $\mathrm{Zn}^{2+}$. The high-resolution $\mathrm{O} 1 \mathrm{~s}$ spectrum (Fig. 2b) presents an asymmetric peak which is resolved into two components at $\sim 529.9 \mathrm{eV}$ and $531.5 \mathrm{eV}$. The peak at $531.5 \mathrm{eV}$ is related to the oxygen species $\left(\mathrm{O}_{\mathrm{x}}^{-}\right)$adsorbed on $\mathrm{ZnO}$ surface, whereas the peak at $529.9 \mathrm{eV}$ can be assigned to the lattice oxygen $\left(\mathrm{O}^{2-}\right)$ in $\mathrm{ZnO}$.

The response to $100 \mathrm{ppm}$ of butanone as a function of the sensor operating temperature was evaluated, as displayed in Fig. $3 a$. The sensor presented the highest response of 29.4 at $400{ }^{\circ} \mathrm{C}$. When the temperature is higher than $400^{\circ} \mathrm{C}$, the butanone molecules begin to desorb of the $\mathrm{ZnO}$ surface before the reaction with the adsorbed oxygen species, which causes a reduction of the butanone response [14]. Thus, the optimum operating temperature of $400{ }^{\circ} \mathrm{C}$ was used to further evaluate the VOC-sensing properties. To study the sensor selectivity, the responses to $100 \mathrm{ppm}$ of several VOCs at $400{ }^{\circ} \mathrm{C}$ were evaluated (Fig. 3b). Clearly, the bicone-like $\mathrm{ZnO}$ structure presents the highest response to butanone, which was about 2.4-19.4 times higher than that of other VOCs. This result suggests the excellent selectivity of bicone-like $\mathrm{ZnO}$ structure to butanone.

The dynamic response curve to different butanone concentrations is shown in Fig. 3c. The sensing response increased with increasing the butanone concentration from 2 to $100 \mathrm{ppm}$. The
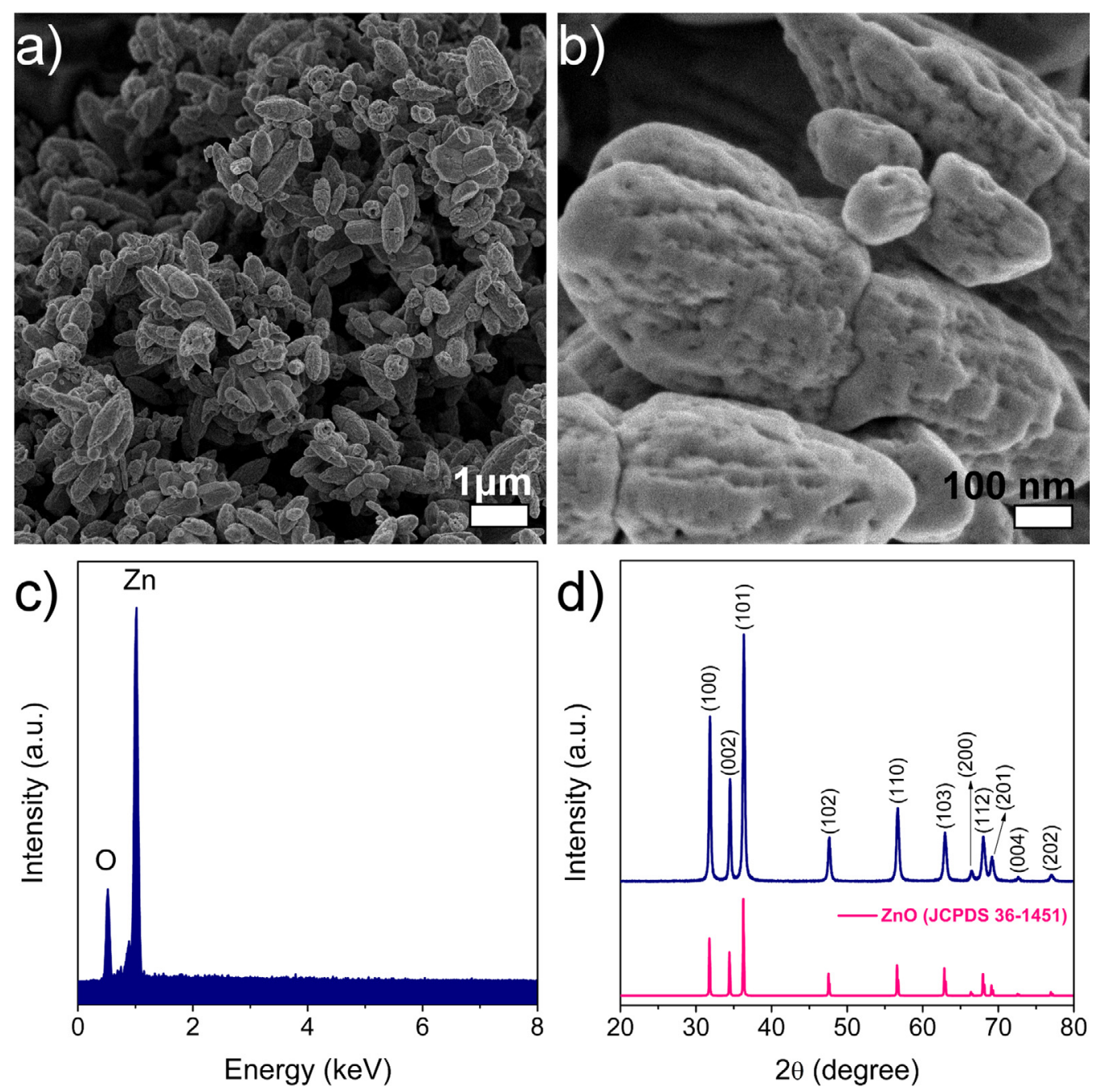

Fig. 1. (a,b) FESEM images in different magnifications, (c) EDS spectra, (d) XRD pattern of bicone-like ZnO structure. 

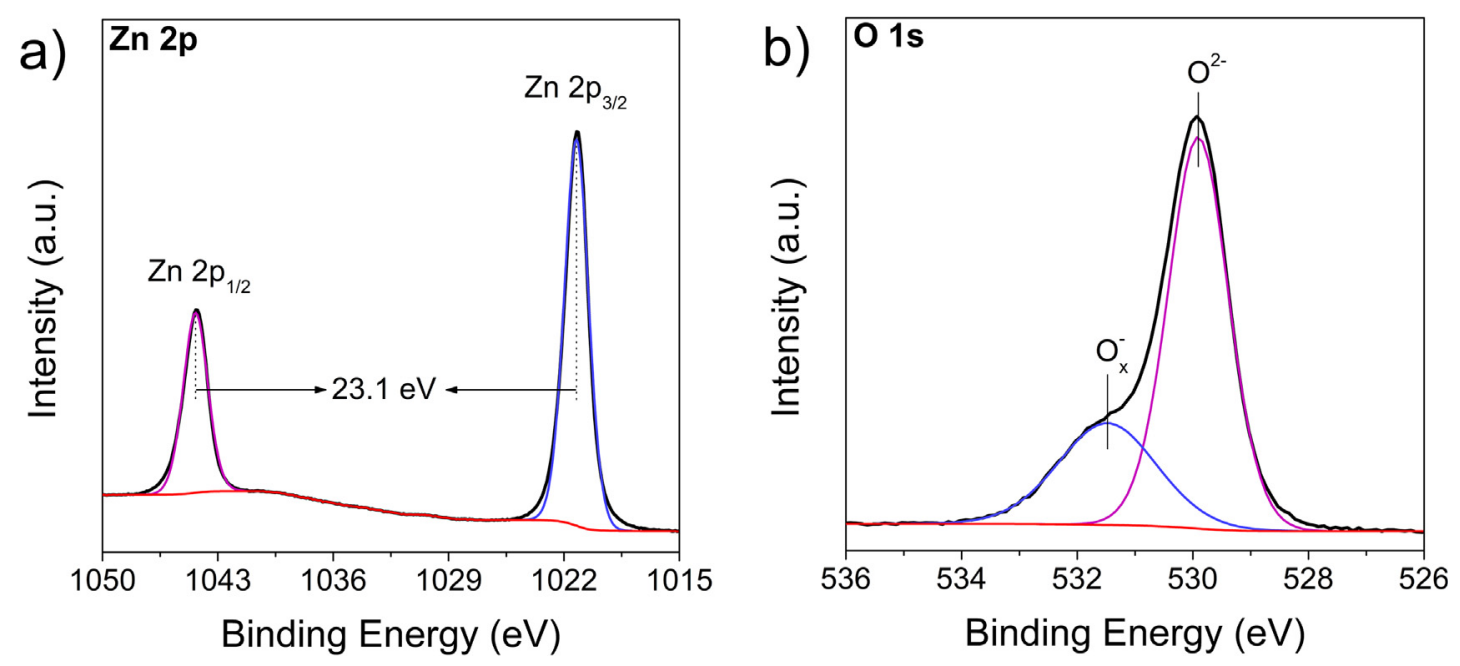

Fig. 2. High-resolution XPS spectrum of (a) Zn 2p and (c) 0 1s.

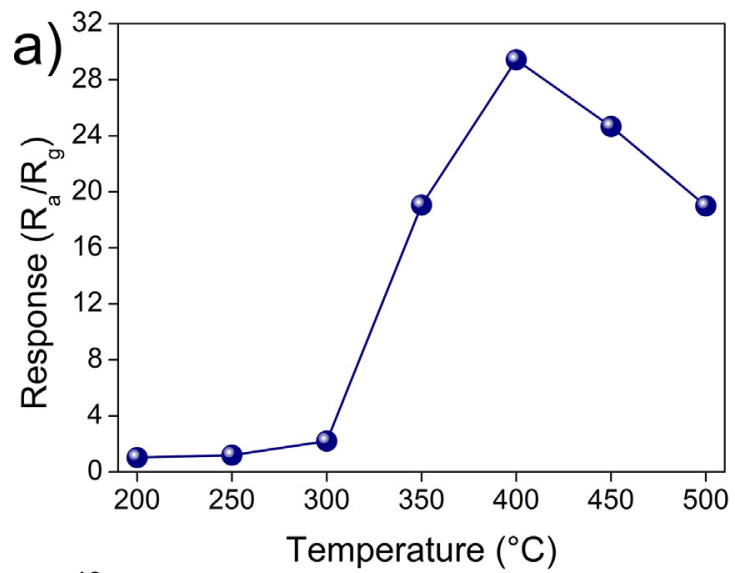

b)
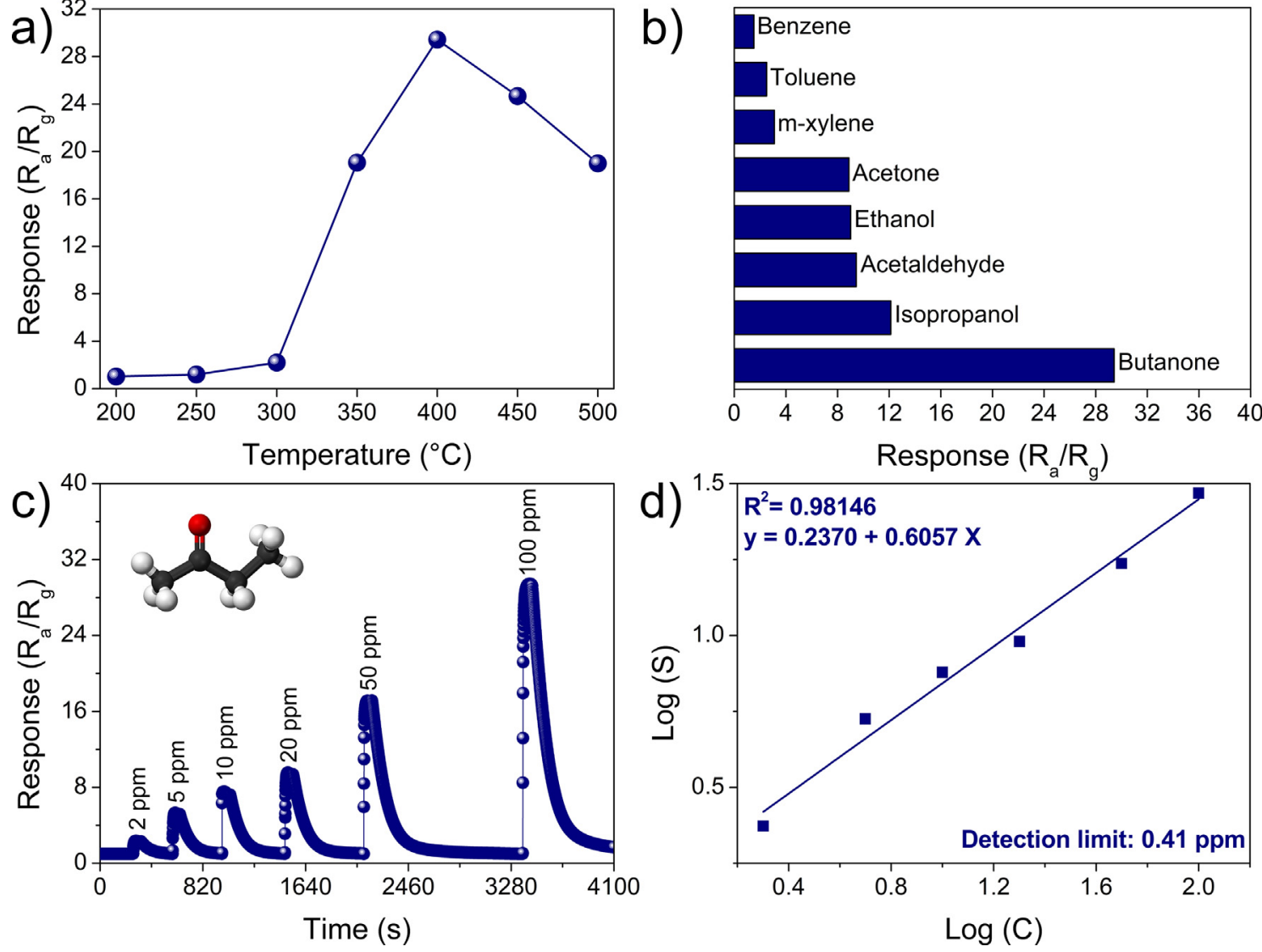

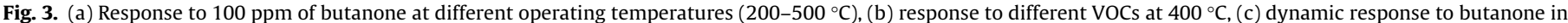
the concentration range of $2-100 \mathrm{ppm}$ at $400^{\circ} \mathrm{C}$, (d) logarithm of the butanone response (S) versus logarithm of butanone concentration (C).

response reached a value of $\sim 2.4$ when the concentration of butanone was $2 \mathrm{ppm}$, indicating an excellent sensitivity. Fig. 3d shows the di-logarithm graphic of the butanone response (S) and butanone concentration (C). The extension of the fit line revealed that the detection limit for butanone is $\sim 0.41 \mathrm{ppm}$, further confirming the high sensitivity of the sensor.

When $\mathrm{ZnO}$ is exposed to air, oxygen molecules can adsorb on its surface and ionize in the form of $\mathrm{O}_{2}^{-}, \mathrm{O}^{-}$or $\mathrm{O}^{2-}[15]$. This process occurs due to the electron transfer from the conduction band
(CB) of $\mathrm{ZnO}$, which creates a depletion layer near the surface. After bicone-like $\mathrm{ZnO}$ sensor is exposed to butanone (a reducing vapor), butanone molecules react with the ionosorbed oxygen species, releasing the electrons back to the $\mathrm{CB}$ of $\mathrm{ZnO}$. Consequently, the depletion layer and the electrical resistance decrease. In this study, $\mathrm{ZnO}$ presented a high butanone response, which can be attributed to the unique bicone-like structure, because each bicone-like structure is composed of assembled nanoparticles, creating more actives sites for gas reaction. 


\section{Conclusion}

In summary, bicone-like $\mathrm{ZnO}$ structure, which are constructed of nanoparticles, have been successfully prepared by a simple template-free MAH method. When applied as a VOC sensor, bicone-like $\mathrm{ZnO}$ structure presented an excellent butanonesensing performance in terms of selectivity, sensitivity, and detection limit, which may be related to its unique morphology. Therefore, bicone-like $\mathrm{ZnO}$ structure is a promising material for highperformance butanone sensors.

\section{Acknowledgments}

The authors thank São Paulo Research Foundation - FAPESP (2017/01267-1, 2016/22219-2, 2016/25267-8, 2016/04371-1, 2013/23886-4) and National Council for Scientific and Technological Development - CNPq (444926/2014-3). FESEM and XPS facilities were provided by LMA/IQ/Unesp and LNNano/CNPEM (proposal 21594), respectively.

\section{References}

[1] C. Wang, X. Cui, J. Liu, X. Zhou, X. Cheng, P. Sun, X. Hu, X. Li, J. Zheng, G. Lu, Design of superior ethanol gas sensor based on Al-doped $\mathrm{NiO}$ nanorod-flowers, ACS Sens. 1 (2015) 131-136.

[2] D. Jiang, W. Wei, F. Li, Y. Li, C. Liu, D. Sun, C. Feng, S. Ruan, Xylene gas sensor based on $\alpha-\mathrm{MoO}_{3} / \alpha-\mathrm{Fe}_{2} \mathrm{O}_{3}$ heterostructure with high response and low operating temperature, RSC Adv. 5 (2015) 39442-39448.

[3] S.-J. Choi, B.-H. Jang, S.-J. Lee, B.K. Min, A. Rothschild, I.-D. Kim, Selective detection of acetone and hydrogen sulfide for the diagnosis of diabetes and halitosis using $\mathrm{SnO}_{2}$ nanofibers functionalized with reduced graphene oxide nanosheets, ACS Appl. Mater. Interfaces. 6 (2014) 2588-2597.
[4] T. Ligor, J. Szeliga, M. Jackowski, B. Buszewski, Preliminary study of volatile organic compounds from breath and stomach tissue by means of solid phase microextraction and gas chromatography-mass spectrometry, J. Breath Res. 1 (2007) 16001.

[5] J. Shin, S.-J.J Choi, I. Lee, D.-Y.Y. Youn, C.O. Park, J-H.H. Lee, H.L. Tuller, I-D.D. Kim, Thin-wall assembled $\mathrm{SnO}_{2}$ fibers functionalized by catalytic Pt nanoparticles and their superior exhaled-breath-sensing properties for the diagnosis of diabetes, Adv. Funct. Mater. 23 (2013) 2357-2367.

[6] S.-J.J. Choi, I. Lee, B.-H.H. Jang, D.-Y.Y. Youn, W.-H.H. Ryu, C.O. Park, I.-D.D. Kim, Selective diagnosis of diabetes using Pt-functionalized $\mathrm{WO}_{3}$ hemitube networks as a sensing layer of acetone in exhaled breath, Anal. Chem. 85 (2013) 1792-1796.

[7] C. Dong, X. Liu, X. Xiao, G. Chen, Y. Wang, I. Djerdj, Combustion synthesis of porous Pt-functionalized $\mathrm{SnO}_{2}$ sheets for isopropanol gas detection with a significant enhancement in response, J. Mater. Chem. A. 2 (2014) 2008920095.

[8] J. Zhang, Z. Qin, D. Zeng, C. Xie, Metal-oxide-semiconductor based gas sensors: screening, preparation, and integration, Phys. Chem. Chem. Phys. 19 (2017) 6313-6329.

[9] B.A. Vessalli, C.A. Zito, T.M. Perfecto, D.P. Volanti, T. Mazon, ZnO nanorods/graphene oxide sheets prepared by chemical bath deposition for volatile organic compounds detection, J. Alloys Compd. 696 (2017) 996-1003.

[10] L. Zhu, W. Zeng, A novel coral rock-like ZnO and its gas sensing, Mater. Lett. 209 (2017) 244-246.

[11] G.K. Mani, J.B.B. Rayappan, ZnO nanoarchitectures: Ultrahigh sensitive room temperature acetaldehyde sensor, Sens. Actuators, B. 223 (2016) 343-351.

[12] X. Xing, X. Xiao, L. Wang, Y. Wang, Highly sensitive formaldehyde gas sensor based on hierarchically porous Ag-loaded $\mathrm{ZnO}$ heterojunction nanocomposites, Sens. Actuators, B. 247 (2017) 797-806.

[13] W. Li, H. Xu, T. Zhai, H. Yu, Q. Xu, X. Song, J. Wang, B. Cao, High-sensitivity, high-selectivity, and fast-recovery-speed triethylamine sensor based on ZnO micropyramids prepared by molten salt growth method, J. Alloys Compd. 695 (2017) 2930-2936.

[14] Z. Wang, J. Xue, D. Han, F. Gu, Controllable defect redistribution of ZnO nanopyramids with exposed $\{1011\}$ facets for enhanced gas sensing performance, ACS Appl. Mater. Interfaces 7 (2015) 308-317.

[15] A. Gurlo, Interplay between $\mathrm{O}_{2}$ and $\mathrm{SnO}_{2}$ : oxygen ionosorption and spectroscopic evidence for adsorbed oxygen, ChemPhysChem 7 (2006) 2041-2052. 